\title{
High Gain Array of Monopoles-Coupled Antennas for Wireless Applications
}

\author{
Ahmad El Sayed Ahmad, ${ }^{1}$ Marc Thevenot, ${ }^{2}$ Jean-Marie Floc'h, ${ }^{1}$ and Mohamad Mantash ${ }^{1}$ \\ ${ }^{1}$ IETR, CNRS, UMR 6164, 20 Avenue des Buttes de Coesmes, 35043 Rennes, France \\ ${ }^{2}$ XLIM, CNRS, UMR 6172, 123 Avenue Albert Thomas, 87060 Limoges, France
}

Correspondence should be addressed to Ahmad El Sayed Ahmad, ahmad-khoder.el-sayed-ahmad@insa-rennes.fr

Received 7 June 2012; Revised 27 September 2012; Accepted 11 October 2012

Academic Editor: Huanhuan Gu

Copyright (C) 2012 Ahmad El Sayed Ahmad et al. This is an open access article distributed under the Creative Commons Attribution License, which permits unrestricted use, distribution, and reproduction in any medium, provided the original work is properly cited.

\begin{abstract}
An array of monopole antennas over a ground plane that radiates a directive lobe in the end-fire direction are described in this paper. The design uses the rigorous method described by Drouet et al. 2008 in order to synthesize the radiation through the strong cumulative coupling between the monopoles. A gain higher than $20 \mathrm{~dB}$ was achieved in the end-fire direction over a $4.5 \%$ bandwidth. However, the antenna has been tilted in order to compensate the beam deviation caused by the edge diffraction. A prototype with 12 elements has been manufactured in order to validate the antenna principle and the whole antenna is successfully measured. The prototype was studied with the software CST-Microwave Studio and the feed network has been designed with Agilent ADS.
\end{abstract}

\section{Introduction}

This paper deals with the design of the vehicular antenna that must satisfy some particular requirements. Firstly, this antenna has to be integrated on the roof that induces a lowprofile antenna working over a ground plane. Secondly, an end-fire antenna which radiates toward the horizon must be used to communicate with the base stations. Finally, the antenna gain must be high in order to reduce the number of base stations. The design of an antenna that satisfies all these specifications is very difficult to perform.

Linear monopole arrays are extensively used in many antenna systems due to their simplicity, low cost, polarization purity, reasonable bandwidth, and power-handling capability [1]. However, the strong mutual coupling between neighbored antenna elements also results in radiation patterns and matching degradations. The feed network can also be directly affected. It has been theoretically demonstrated that mutual coupling effects on radiation patterns can be reduced with appropriate loads [2-5].

The aim of this paper is to design a linear array of monopoles by managing the coupling. Moreover, the antenna design must be robust and easy to manufacture in order to be integrated on a vehicle roof, and, thus, to undergo outdoor conditions such as rain and wind.

In the first part, the global design method will be briefly explained. Then, the principle, the design, and the performances of a linear array of 12 monopoles will be given. In the second part, an array of $4 \times 12$ monopoles fed by a feed network will be described. The last section discusses the design of $4 \times 12$ monopoles that would be compared to a Yagi antenna.

\section{Basic Structure}

2.1. Principle. The basic structure is composed of twelve monopoles and a feed network. The strong interactions between the monopoles need to design the feed network with a great accuracy in order to optimize the directivity.

The objective consists of the determination of the impedance matching and the incident power to reach both the objective radiation pattern and the best matching for the monopole array.

We employ the method described in [6] for the design of the array antenna with strong coupling: by using CSTMicrowave studio we compute the $[S]$ matrix and the 12 
radiation patterns when the 12 monopoles are successively fed. These radiation patterns are $\phi_{1}$ to $\phi_{12}(1)$. An objective radiation pattern $\phi_{\mathrm{obj}}$ is proposed. This objective radiation pattern can be the linear combination of the radiation pattern of one monopole on its limited ground plane multiplied by an array factor (2). In this relation, $d$ is the distance between each monopole and $\varphi_{i}$ is the phase of the ith monopole.

Equation (1) provides the weights that must be applied to the monopoles' radiation pattern. Equation (3) leads to the antenna impedances to be considered as a reference $\left(Z_{\text {ref }}\right)$ in order to reach the matching and (4) gives the input waves that the feed network must achieve:

$$
\begin{gathered}
{\left[\phi_{1} \phi_{2} \cdots \phi_{i}\right]\left(\begin{array}{c}
\beta_{1} \\
\beta_{2} \\
\vdots \\
\beta_{i}
\end{array}\right) \approx \phi_{\text {obj }} \quad \text { with } 1 \leq i \leq 12} \\
\phi_{\text {obj }}=\sum_{i=1}^{12} \exp \left[-\left(k_{0} \cdot d \cdot \sin (\theta)+\varphi_{i}\right) \cdot i\right] \cdot \phi_{\text {monopole }} \\
Z_{\text {ref } \_i_{1}}=\left(\frac{50 \cdot[I+S](\beta)}{[I-S](\beta)}\right)^{*} \\
a_{i}=\sqrt{50} \cdot[I+S](\beta) \frac{\sqrt{\Re\left(Z_{\text {ref } \_i}\right)}}{Z^{*}{ }_{\text {ref } \_i}}
\end{gathered}
$$

where $\phi_{i}$ is the $i$ th monopole radiation pattern, $\phi_{\text {obj }}$ is the objective radiation pattern, $\beta_{i}$ is the weight that must be applied to the $i$ th monopole radiation pattern, $d$ is the distance between each monopole, $k_{0}=2 \pi / \lambda_{0}\left(\lambda_{0}\right.$ free space wavelengths), $\varphi_{i}$ is the phase shift at $i$ th monopole, and $[S]$ is the coupling matrix.

2.2. Design and Performances of the Array of 12 Monopoles. As explained in Section 1, the application is a communication system that uses the WIMAX protocol between a vehicle and base stations. The objective is to establish a highgain monopole array that radiates a directional beam in the azimuthal plane within the frequency band $5.47 \mathrm{GHz}-$ $5.725 \mathrm{GHz}$. In this section, we propose the complete design of the array of monopoles with its feed network. The optimization frequency is $5.6 \mathrm{GHz}$.

In order to achieve a radiation with a single lobe in the direction of the array alignment, the space between two nearby monopoles must stay lower than $0.5 \lambda_{0}$ : we have chosen $0.45 \lambda_{0}(24.12 \mathrm{~mm})$ for our design. Twelve monopoles are set on a ground plane whose dimensions are $L x=100 \mathrm{~mm}$ and $L y=330 \mathrm{~mm}$ (Figure 1). The monopole lengths are listed into Table 1 (length) and their diameter is $2.53 \mathrm{~mm}$. The connections between the monopoles and the feed network's ports are achieved with $50 \Omega$ coaxial transitions which are drilled through the ground plane (Figure 2 ). The feed network is printed back to the antenna ground plane, onto a $0.508 \mathrm{~mm}$ thick Duroïd 6002 substrate $(\varepsilon r=2.94$, $\operatorname{tg} \delta=0.0012$ ).

The array of monopoles is positioned on a limited ground plane. In the limited ground plane size case, the

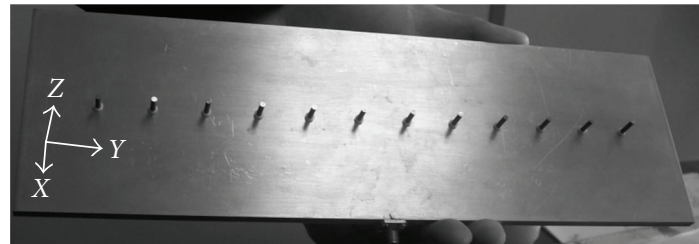

FIGURE 1: The array antenna is composed of 12 monopoles.

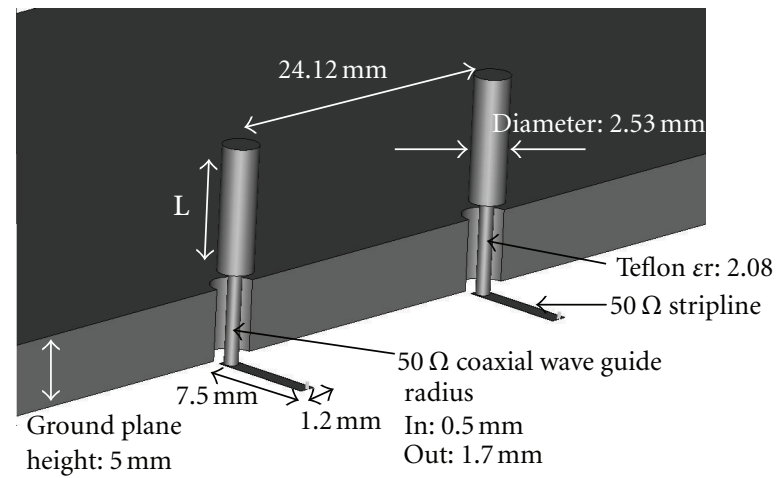

Figure 2: The monopoles are fed by a coaxial guide drilled through the ground.

well-known scattering effects on the ground plane edges alter the radiation pattern [7-9] (Figure 3). First of all, the interferences induce maxima and minima field on the radiation pattern. Their angular position is obviously related to the ground plane size. Then, we can observe the classic beam deviation in the elevation plane, which is caused by the scattering on the edges of the limited ground plane, since the main beam direction does not coincide with the horizon. To achieve the objective radiation pattern (we defined an angle $\theta=75^{\circ}$ ), we apply the array factor (2) with

$$
k_{0} \cdot d \cdot \sin (\theta)+\varphi_{i}=m \cdot 2 \cdot \pi \Longrightarrow \varphi_{i}=203^{\circ} .
$$

It should to be stressed that these results are approximations since the analysis considers the monopoles do not interfere with each other. The radiation pattern illustrated in Figure 4 (monopole $x$-array factor) can be used as the objective radiation pattern $\phi_{\text {obj }}$. In the next step, we have used CST Microwave studio to achieve the full-wave analysis of the whole antenna structure. As an example, only 3 monopole radiation patterns are plotted in Figure 3.

According to (1), the weights $\beta$ are deduced and written in Table 1. Thus, Figure 4 points out the resemblance between the objective radiation pattern and the linear combination of the radiation patterns of monopoles weighted by the coefficients $\beta$.

Figures 5 and 6 show the scattering matrix of the monopole antenna. Regarding Figure 6, these interactions should not be omitted when connecting the array monopoles with the feed network. The coupling between nearby monopoles is greater than $-13 \mathrm{~dB}$.

The optimum weights $\left(a_{i}\right)$ and the input impedances $\left(Z_{\text {ref_i }_{-}}\right)$which simultaneously perform the objective radiation 


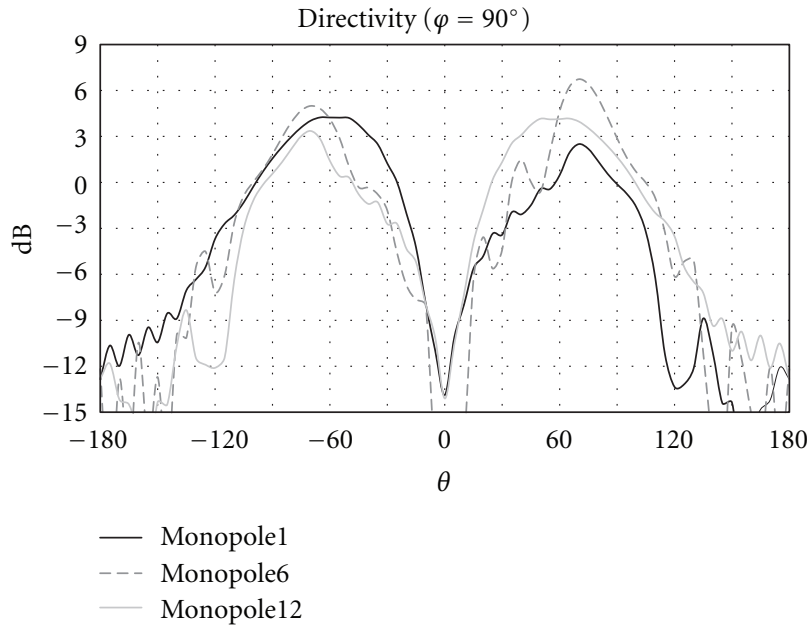

FIGURE 3: Radiation patterns of monopoles when the monopoles are successively fed.

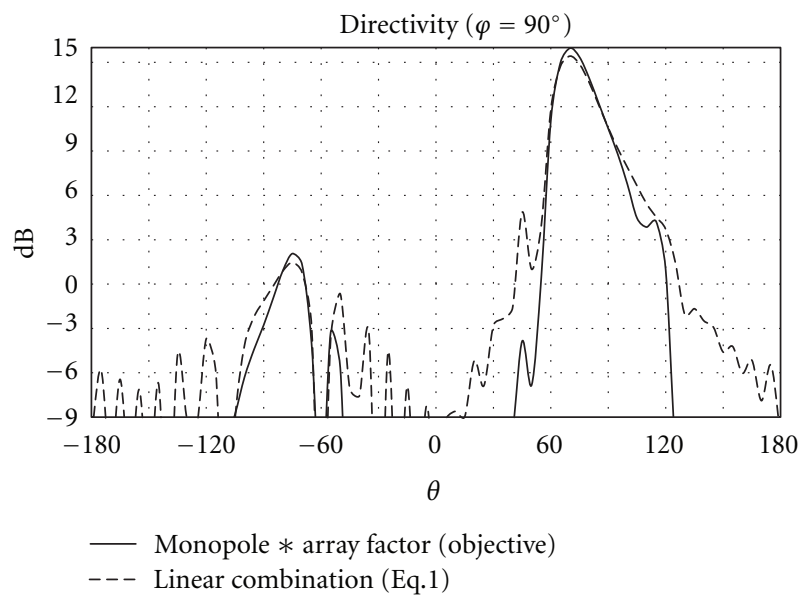

FIGURE 4: Comparison of the objective radiation pattern (radiation pattern of monopole $x$-array factor) with the linear combination of the radiation patterns of monopoles $(f=5.6 \mathrm{GHz})$.

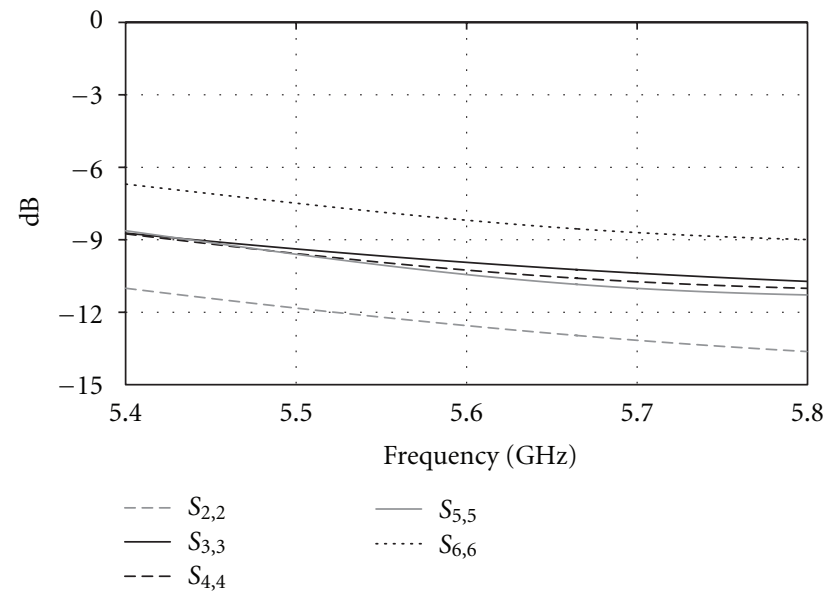

FIGURE 5: Some $S_{i i}$ parameters of the array of monopoles.

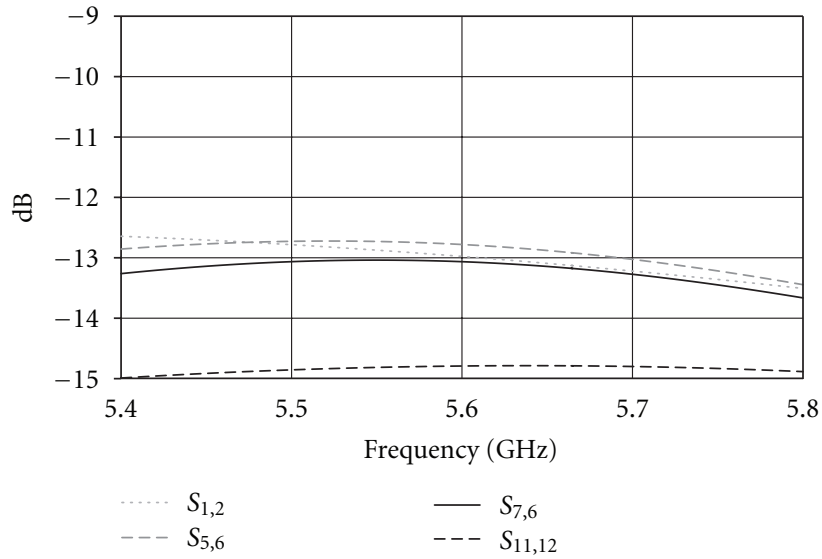

FIgURE 6: Some $S_{i j}$ parameters of the array of monopoles.

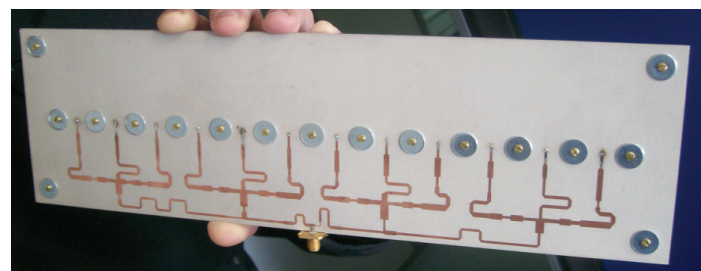

FIGURE 7: The feed network is designed to maximize the efficiency of the strongly coupled monopoles.

and the matching of all the feeding ports can be calculated using (3), (4), the scattering matrix $[S]$, and the $\beta$ vector. These values are given in Table 1 (columns 4 and 5) with the optimized monopole lengths. These have been set to comply with the different impedance values resulting from the synthesis procedure and to minimize the feed distribution network complexity.

The design of the microstrip feed network has been made with the Agilent ADS software in order to perform the weights and the impedance matching specified in Table 1. The realized feed network is shown in Figure 7.

In order to perform the numerical validation, the monopole simulation and the feed network design are numerically connected together. Using the CST software, this entire structure simulation provides the performances of the whole array antenna (the 12 monopoles and the feed network). The radiation pattern, the gain, and the return loss are computed. Figure 8 plots the radiation pattern in the plane phi $=90^{\circ}$. This is the plane which is parallel to the array alignment. We can observe that the entire structure simulation agrees very well with the objective radiation pattern (linear combination of the radiation patterns of the monopoles). So, the feed network operates properly through the couplings. Figure 9 presents the radiation pattern in 3D at $5.6 \mathrm{GHz}$; the maximum simulated directivity is $15.6 \mathrm{~dB}$. The main beam direction does not coincide with the horizon $\left(\theta=90^{\circ}\right)$; it will be necessary to compensate this deviation by an inclination of the whole antenna. Indeed, it is essential for our application that the maximum gain is radiated in the base stations direction. 
TABLE 1: Normalized incident waves and reference impedances which optimize the efficiency of the array antenna for a specified radiation pattern $(5.6 \mathrm{GHz})$.

\begin{tabular}{|c|c|c|c|c|}
\hline \multirow[t]{2}{*}{ Monopoles } & \multirow{2}{*}{$\begin{array}{l}\text { Length } \\
(\mathrm{mm})\end{array}$} & \multirow[t]{2}{*}{$\beta$ (= weights for the coupled radiation patterns) } & \multicolumn{2}{|c|}{$\begin{array}{l}\text { Normalized incident waves }\left(a_{i}\right) \text { and antenna } \\
\text { impedances }\left(Z_{\mathrm{ref}_{i} i}\right) \text { that optimize the efficiency }\end{array}$} \\
\hline & & & $a_{i}$ & $Z_{\text {ref } \_i}$ \\
\hline 1 & 10.8 & $0.286 \cdot \exp ^{\left(-j^{*} 48^{\circ}\right)}$ & $0.288 \cdot \exp ^{\left(-j^{*} 49^{\circ}\right)}$ & $29+j^{*} 13$ \\
\hline 2 & 9.8 & $0.293 \cdot \exp ^{\left(j^{*} 155^{\circ}\right)}$ & $0.30 \cdot \exp ^{\left(j^{*} 152^{\circ}\right)}$ & $32+j^{*} 10$ \\
\hline 3 & 9.3 & $0.279 \cdot \exp ^{\left(-j^{*} 3^{\circ}\right)}$ & $0.286 \cdot \exp ^{\left(-j^{*} 6^{\circ}\right)}$ & $31+j^{*} 9$ \\
\hline 4 & 9.3 & $0.284 \cdot \exp ^{\left(-j^{*} 155^{\circ}\right)}$ & $0.291 \cdot \exp ^{\left(-j^{*} 146^{\circ}\right)}$ & $30+j^{*} 1$ \\
\hline 5 & 9.3 & $0.284 \cdot \exp ^{\left(j * 47^{\circ}\right)}$ & $0.287 \cdot \exp ^{\left(j^{*} 35^{\circ}\right)}$ & $27-j^{*} 2$ \\
\hline 6 & 8.8 & $0.280 \cdot \exp ^{\left(-j^{*} 120^{\circ}\right)}$ & $0.283 \cdot \exp ^{\left(-j^{*} 124^{\circ}\right)}$ & $29+j * 7.5$ \\
\hline 7 & 8.8 & $0.266 \cdot \exp ^{\left(j^{*} 82^{\circ}\right)}$ & $0.262 \cdot \exp ^{\left(j^{*} 79^{\circ}\right)}$ & $24+j^{*} 8.5$ \\
\hline 8 & 8.8 & $0.264 \cdot \exp ^{\left(-j^{*} 74^{\circ}\right)}$ & $0.251 \cdot \exp ^{\left(-j^{*} 77^{\circ}\right)}$ & $20+j^{*} 8.5$ \\
\hline 9 & 8.8 & $0.254 \cdot \exp ^{\left(j^{*} 131^{\circ}\right)}$ & $0.227 \cdot \exp ^{\left(j^{*} 124^{\circ}\right)}$ & $15+j^{*} 3$ \\
\hline 10 & 8.8 & $0.275 \cdot \exp ^{\left(-j^{*} 24^{\circ}\right)}$ & $0.257 \cdot \exp ^{\left(-j^{*} 35^{\circ}\right)}$ & $18-j^{*} 1.5$ \\
\hline 11 & 8.8 & $0.319 \cdot \exp ^{\left(j^{*} 180^{\circ}\right)}$ & $0.323 \cdot \exp ^{\left(j^{*} 165^{\circ}\right)}$ & $28-j^{*} 7$ \\
\hline 12 & 8.3 & $0.361 \cdot \exp ^{\left(j^{*} 0^{\circ}\right)}$ & $0.377 \cdot \exp ^{\left(j^{*} 0^{\circ}\right)}$ & $48+j^{*} 17$ \\
\hline
\end{tabular}

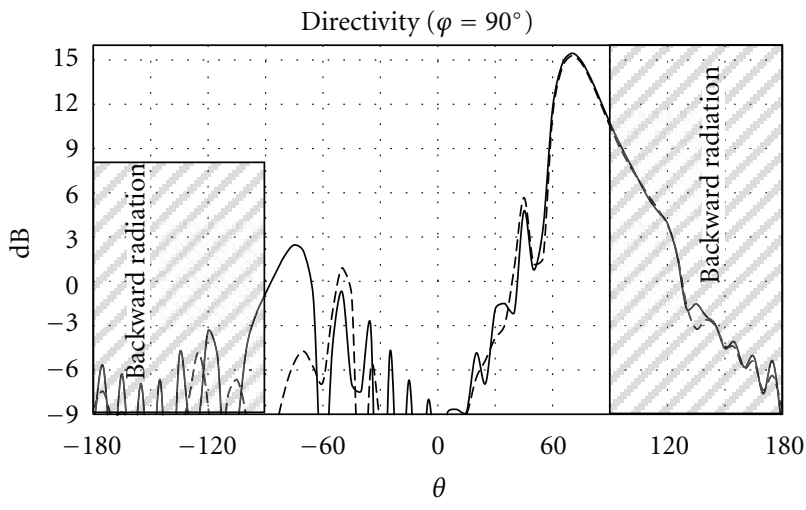

Linear combination of the element pattern (Eq.1)
- $\begin{aligned} & \text { Entire structure simulation (feed network } \\ & \text { connected with the monopoles) }\end{aligned}$

FIGURE 8: Simulated radiation pattern (directivity) comparison at $f=5.6 \mathrm{GHz}$.

The return loss at the input of the feed network is plotted in Figure 10 (simulation). The level is lower than $-15 \mathrm{~dB}$ over the operating frequency bandwidth. This numerical validation shows that the radiation pattern is successfully synthesized as well as the impedance matching of every antenna port through the couplings. Although the feed network has been optimized to deal with the antenna couplings at $5.6 \mathrm{GHz}$, we have evaluated the performances of the entire structure (12 monopoles connected with the feed network) from $5.47 \mathrm{GHz}$ to $5.725 \mathrm{GHz}$.

The antenna gain is $14.7 \mathrm{~dB}$ over the $5.47 \mathrm{GHz}-$ $5.725 \mathrm{GHz}$ operating bandwidth (Figure 11). The directivity and the gain difference are mainly due to the dielectric losses in the strip line circuit.

2.3. Measurements. The array of monopoles and the feed network were manufactured (Figures 1 and 7 ). The feed

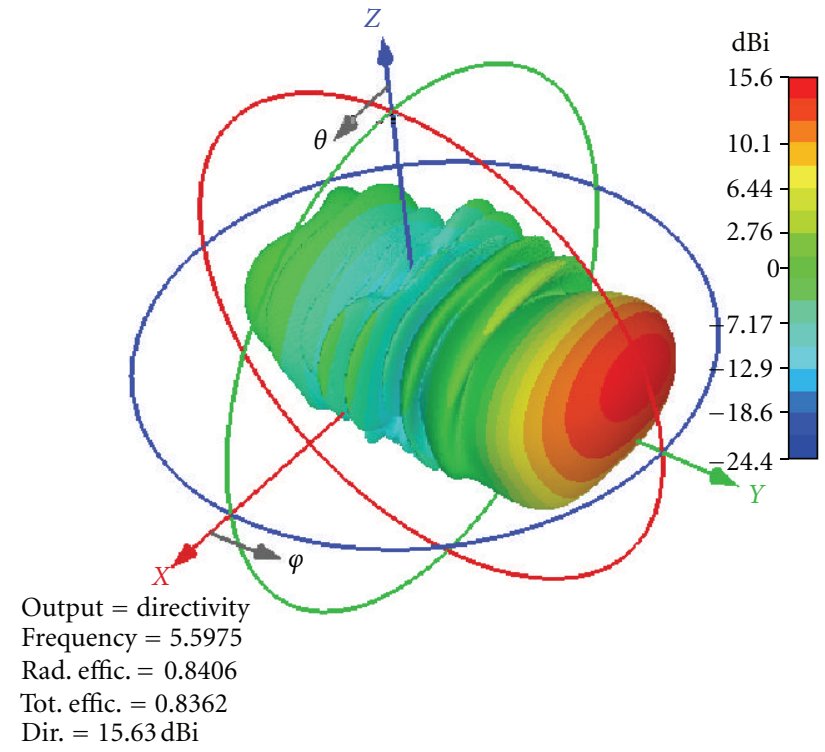

FIGURE 9: Simulated radiation pattern in 3D (directivity) at $f=$ $5.6 \mathrm{GHz}$.

network is glued back to the ground plane and screws were added to secure the RF contacts. We have checked that interactions between the screws and the circuit are negligible. An SMA connector is at the input port.

Measurements were achieved in an anechoic chamber. The return loss of the tested antenna is in Figure 10 (measurement). This measurement is compared with the simulation: both $S_{11}$ are close to $-15 \mathrm{~dB}$ over the operating frequency bandwidth. A slight discrepancy of $50 \mathrm{MHz}$ can be observed compared to the simulation, but it represents only $0.9 \%$ of the frequency shift that can be due to the mesh accuracy during simulation or manufacture tolerance. Figure 12 compares the measured radiation pattern with the 


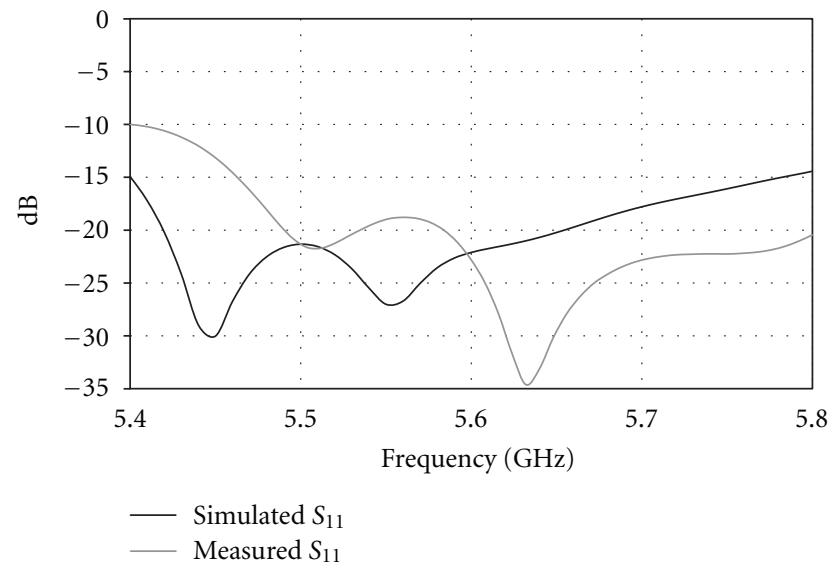

FIGURE 10: Comparison of the $S_{11}$ of the entire structure simulation (12 monopoles connected with the feed network) with the realized structure.

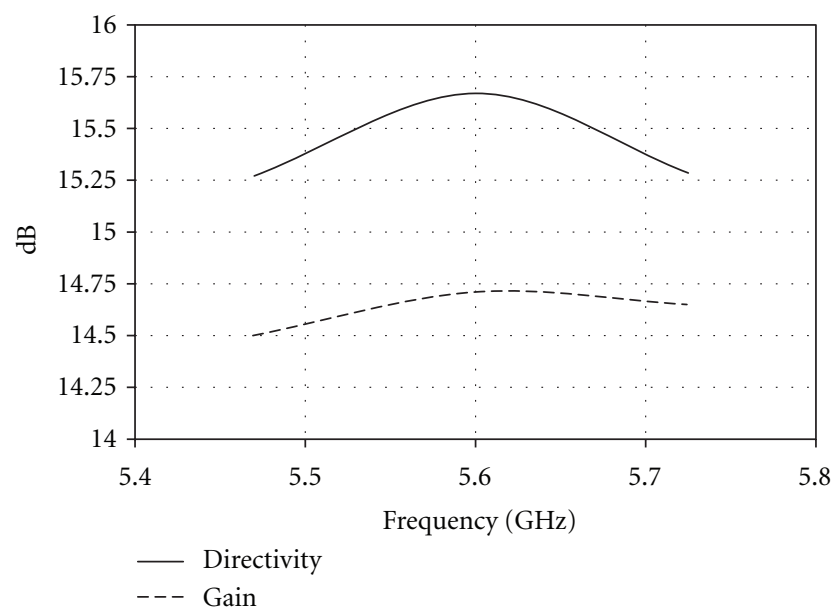

Figure 11: Directivity and gain versus the frequency $\left(\theta=75^{\circ}, \varphi=\right.$ $\left.90^{\circ}\right)$.

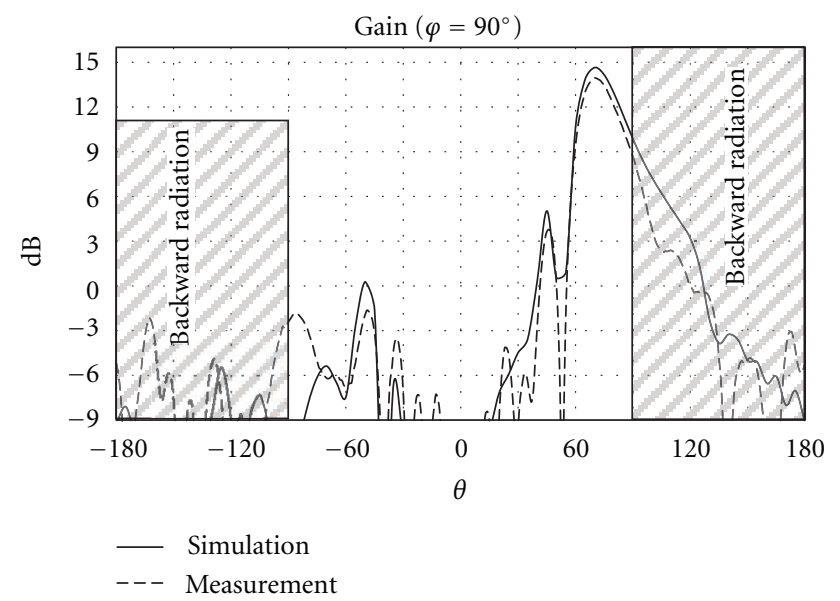

FIGURE 12: Comparison of the objective radiation pattern simulated with the radiation pattern measured at $f=5.6 \mathrm{GHz}$.

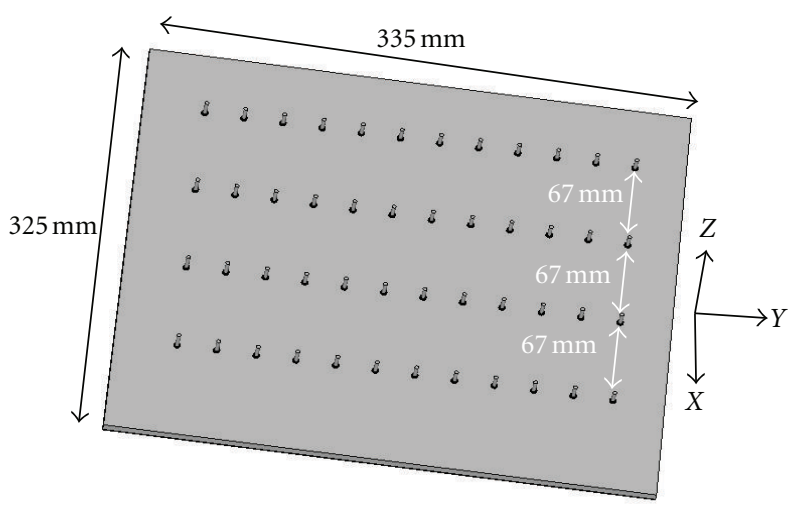

Figure 13: Array of $4 \times 12$ monopoles designed with CST Mws separated by $0.45 \lambda_{0}$ along [oy] and $1.25 \lambda_{0}$ along [ox].

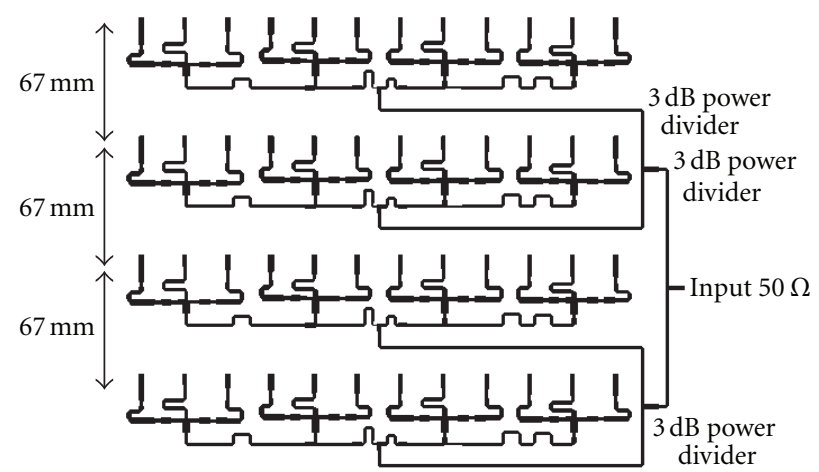

FIgURE 14: The feed network is designed to the four-subarray antenna.

theoretical one over $360^{\circ}$ in the plane of the array alignment $\left(\varphi=90^{\circ}\right)$. The measured gain agrees very well with the prediction. We can conclude that the design is reliable. The feed network operates properly through the couplings. The differences between the simulated and the measured gains are lower than $0.5 \mathrm{~dB}$. Metallic losses in the feed network and the uncertainty accuracy of our anechoic chamber can be responsible for this discard.

\section{2D Array of $4 \times 12$ Monopoles (4 Subarrays)}

The well-behaved experimental results validate the principle of the 12-monopole linear array. The linear array of twelve monopoles (along [oy]) provided a gain of $14.7 \mathrm{~dB}$ at $5.6 \mathrm{GHz}$. Figure 9 shows that the radiation pattern contains low side lobes in the perpendicular plane [ox] to the array of monopole plane alignment [oy]. In order to increase the gain, a 2D array of $4 \times 12$ monopoles was designed (Figure 13). Four sub-arrays, where each of them is described in Section 2, have been used to make the 48-monopole array. Therefore, the 4 sub-arrays are $1.25 \lambda_{0}$ spaced out in order to avoid the interferences in these directions. Obviously, these sub-arrays alignment allow the constructive interference and so increase the gain in the end-fire direction. 


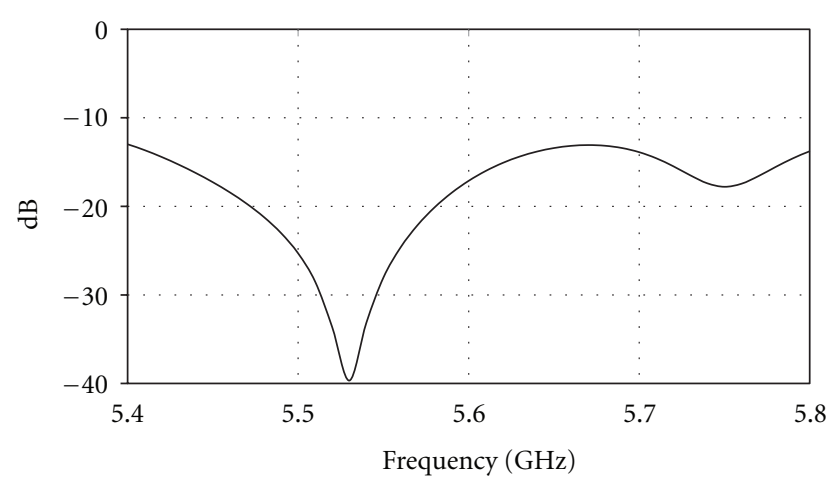

Figure 15: Modulus of $S_{11}$.

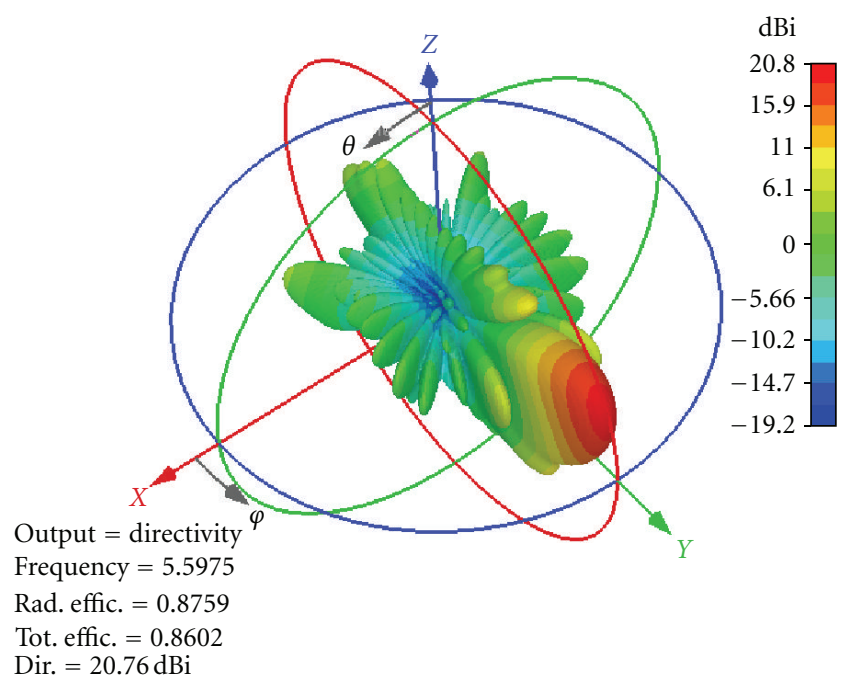

FIgURE 16: Simulated radiation pattern in 3D (directivity) of $4 \times 12$ monopoles at $5.6 \mathrm{GHz}$.

$3 \mathrm{~dB}$ power dividers have been designed to connect the feed networks. $1.25 \lambda_{0}(67 \mathrm{~mm})$ is sufficient in order to avoid the interferences between the lines of the feed network. The corresponding layout of the feed network of $4 \times 12$ monopoles is shown in Figure 14. The return loss at the input of the feed network is plotted in Figure 15. The level is lower than $-15 \mathrm{~dB}$ over the operating frequency bandwidth. The $3 \mathrm{D}$ (Figure 16) radiation pattern shows a very directive lobe. A $20.8 \mathrm{~dB}$ maximum directivity is obtained at the end-fire direction. An increase of $5.2 \mathrm{~dB}$ has been obtained compared to the case with a single subarray (12 monopoles) (Figure 9). The antenna gain is $20 \mathrm{~dB}$ over the $5.47 \mathrm{GHz}-5.725 \mathrm{GHz}$ operating bandwidth (Figure 17). The directivity and the gain difference are mainly due to the dielectric losses in the strip line circuit. Indeed, the insertion losses are very low because the antenna reflection coefficient is lower than $-15 \mathrm{~dB}$ over the $5.47 \mathrm{GHz}-5.725 \mathrm{GHz}$ band (Figure 15).

The $4 \times 12$ monopoles are sufficient to have the gain required in the specifications. The antenna was $15^{\circ}$ tilted to give back the main beam deviation caused by the scattering at the ground plane edges.

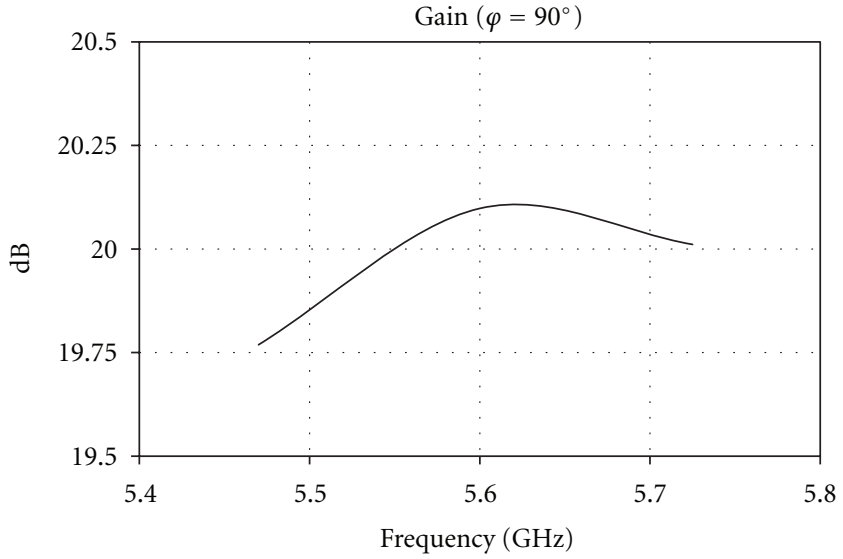

Figure 17: Gain versus the frequency $\left(\theta=75^{\circ}, \varphi=90^{\circ}\right)$.

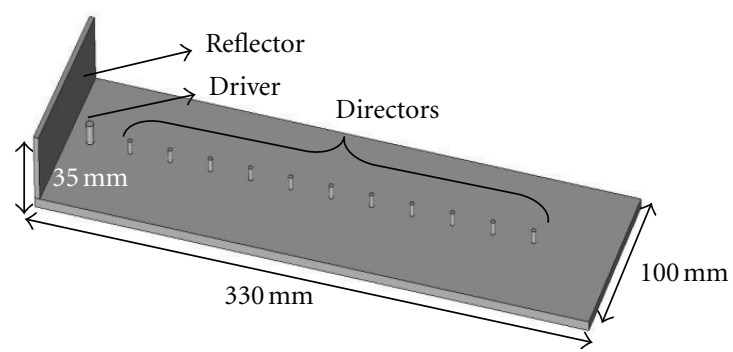

FIgure 18: Proposed Yagi antenna.

\section{Yagi Antenna}

In order to check the interest to develop the complete method for the conception, we have made another antenna. The proposed antenna is a Yagi-Uda antenna.

Yagi antennas of three or more elements are widely used, although a thorough study is lacking today because of the many parameters, each element having three variables, length, spacing, and the diameter of conductor. Almost all multielement Yagis are invariably designed empirically. In [10], Yagi antenna of three elements was presented. It has been shown the gain over a half-wave dipole of a three element Yagi with various director lengths and spacing. This study shows that as the spacing between director and driver decreases, the optimum length of the director increases.

It has been documented in [11-13] that the dimension ratio of the reflector to the driven element can be somewhere between 1.1 and 1.3. The dimension ratio of the director to the driven element can be between 0.8 and 0.95 . The distance between the centers of the reflector and the driven element should be about 0.25 free-space wavelengths, while the separation between the centers of the director and the driven element and the separation between the directors themselves should be between 0.3 and 0.4 free-space wavelengths.

The antenna characteristics such as gain, front-to-back ratio, beamwidth, and center frequency can be altered by changing the length of the driven element, the length of the parasitic elements, spacing between reflector and dipole, and spacing between director and dipole [14]. 


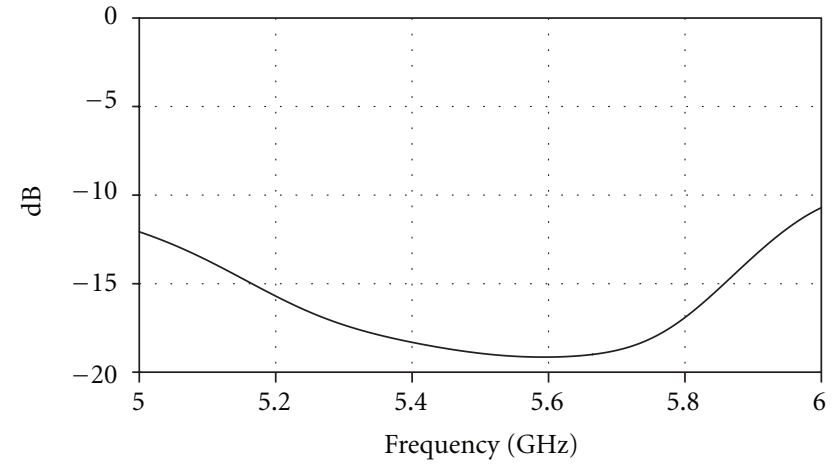

Figure 19: $S_{11}$ of the Yagi antenna.

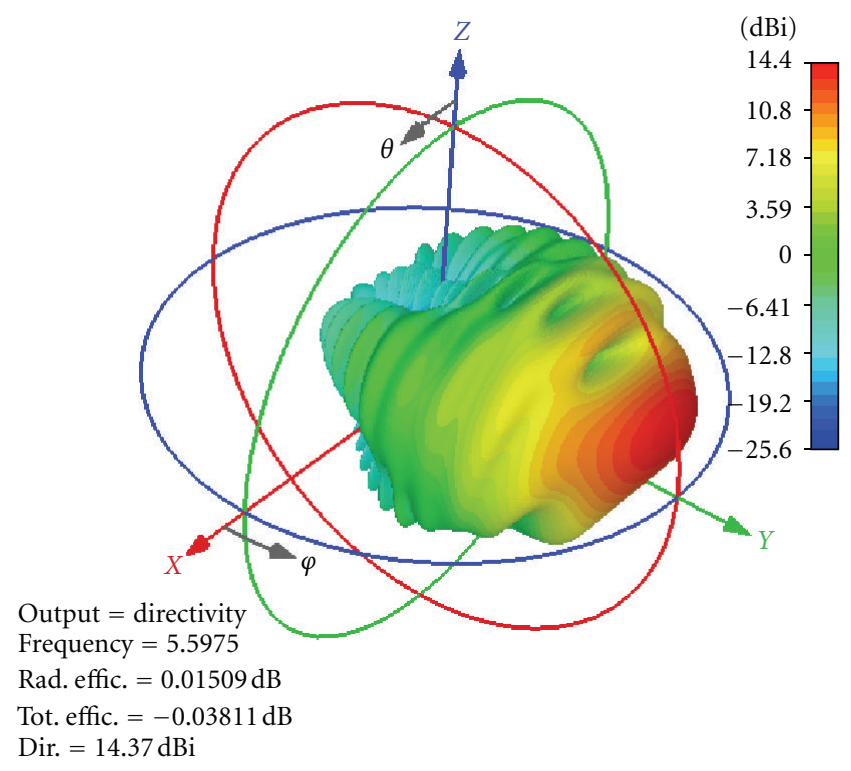

FIgURe 20: Simulated radiation pattern in 3D (directivity) of Yagi antenna at $5.6 \mathrm{GHz}$.

The proposed antenna consists of a monopole as a driven element, a reflector, and eleven directors as shown in Figure 18. To facilitate the design, this antenna is designed using the same size of the prototype described in Section 2. Since our application requires only one high-gain radiation direction, it is proceeded to prohibit the radiation in the half space behind the antenna. The backfire radiation can be avoided with some non excited elements named "reflectors" or with a vertical metallic plane. Intended for simplicity constraints, the second solution is selected. So, the driver monopole must be spaced out of a $\lambda_{0} / 4(13.4 \mathrm{~mm})$ distance from the reflector plane. This separation allows a constructive interference between the reflected fields and the direct waves. In this case and according to the images theory, the antenna gain should be $3 \mathrm{~dB}$ increased at the end-fire direction.

The separation between the centers of the director and the driven element and the separation between the directors themselves is 0.45 free-space wavelength $(24.12 \mathrm{~mm})$. The director lengths are $6.7 \mathrm{~mm}\left(\lambda_{0} / 8\right)$ and their diameters are

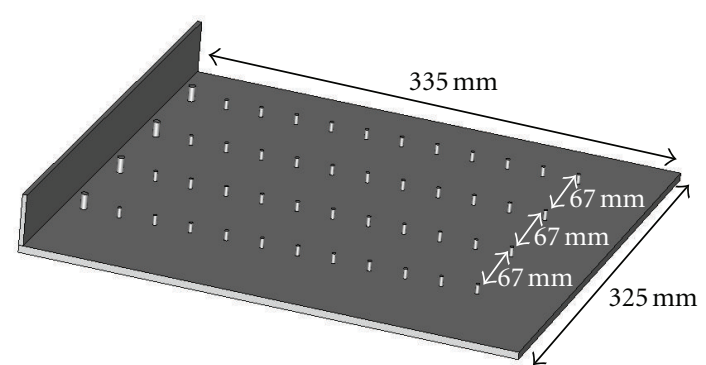

Figure 21: Array of $4 \times$ Yagi antenna.

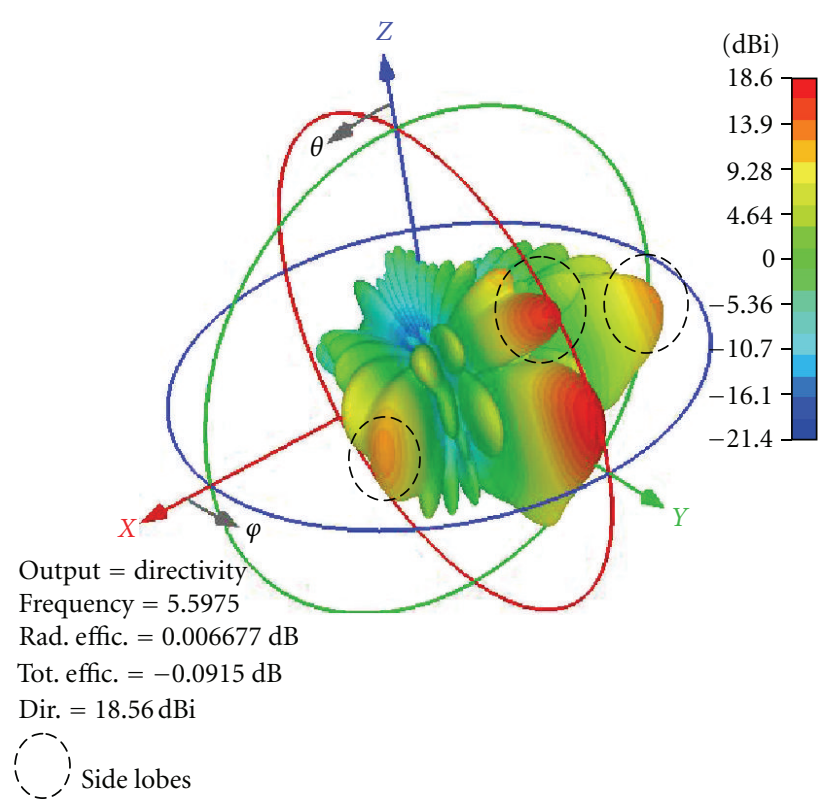

FIGURE 22: Simulated radiation pattern in 3D (directivity) of $4 \times$ Yagi antenna at $5.6 \mathrm{GHz}$.

$2.53 \mathrm{~mm}$. These directors are shortcircuited with the ground plane. The length of the driver monopole is $10.32 \mathrm{~mm}$; its diameter is $4.53 \mathrm{~mm}$.

The yagi antenna is matched to $-18 \mathrm{~dB}$ in simulation over a bandwidth $5.47 \mathrm{GHz}-5.725 \mathrm{GHz}$ (Figure 19). The simulated radiation pattern in $3 \mathrm{D}$ is presented in Figure 20; the maximum directivity is $14.3 \mathrm{~dB}$ at the end-fire direction.

In order to increase the directivity, a $2 \mathrm{D}$ array of $4 \times$ Yagi antenna was designed (Figure 21). The antenna was designed using the same size of the prototype described in Section 3 to make a true comparison between the array of monopole antenna and the Yagi antenna. Figure 22 presents the radiation pattern at $5.6 \mathrm{GHz}$. We obtain a maximum directivity of $18.5 \mathrm{~dB}$. The comparison of radiation in the Cartesian plane between the array of monopoles and the yagi antenna is shown in Figure 23. The radiation pattern is compared versus $\varphi$ at $\theta=75^{\circ}$ (maximum radiation). We can observe the first side lobe level of yagi radiation pattern is around $12 \mathrm{~dB}$; it is $-6 \mathrm{~dB}$ below the main lobe which explains the maximum directivity of yagi antenna is $2.3 \mathrm{~dB}$ lower than the radiation of the monopole array. 


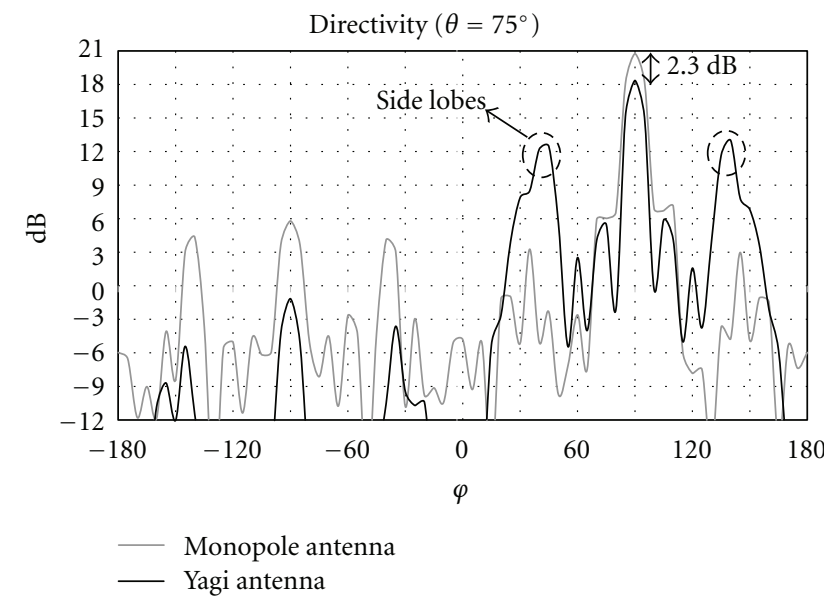

FIGURE 23: Comparison of the radiation pattern of $4 \times 12$ monopoles with the $4 \times$ Yagi array antenna.

The advantages of the monopole antenna compared to the Yagi antenna are

(1) the array of monopole antenna designed in Section 3 does not need to a reflector plane to radiate on the end-fire direction,

(2) the radiation pattern of monopole antenna does not contain significant side lobes levels,

(3) the maximum level of radiation of the monopoles antenna is greater than the yagi,

The disadvantages of the monopole antenna compared to the Yagi antenna is the feed network.

\section{Conclusion}

In this paper, a low-profile antenna with a ground plane has been presented. The purpose was to design a high-gain antenna (single end-fire beam) which must be positioned on a vehicle roof in order to communicate with the far base stations. As a first step, an array of 12 monopoles was designed. In such a structure, the monopoles strongly interact with each other.

In our study, the feed network has been designed to deal with the couplings by considering as a reference the impedances and the input waves that optimize the efficiency of the antenna.

The feed network and the monopole array were manufactured. The whole antenna was successfully tested. The antenna was tilted to give back the main beam deviation caused by the scatterings on the ground plane edges.

As a second step, an array of $4 \times 12$ monopoles has been designed in order to increase the gain. A gain higher than $20 \mathrm{~dB}$ has been achieved over a $4.5 \%$ bandwidth.

Finally, in order to check the interest to develop the complete method for the conception, we have made another antenna. The proposed antenna is a Yagi-Uda antenna. The radiation of this antenna presents high side lobe levels. The maximum radiation on the end fire is lower than the radiation of the monopole array. In conclusion, as the method takes into account couplings, a particular beam pointing with reduced or controlled side lobes can be achieved easily.

\section{References}

[1] B. Tomasic and A. Hessel, "Linear array of coaxially fed monopole elements in a parallel plate waveguide-I: theory," IEEE Transactions on Antennas and Propagation, vol. 36, no. 4, pp. 449-462, 1988.

[2] D. M. Pozar, "The active element pattern," IEEE Transactions on Antennas and Propagation, vol. 42, no. 8, pp. 1176-1178, 1994.

[3] J. P. Daniel and C. Terret, "Mutual coupling between antennas optimization of transistor parameters in active antenna design," IEEE Transactions on Antennas and Propagation, vol. AP-23, no. 4, pp. 513-516, 1975.

[4] A. K. Bhattacharyya, Phased Array Antennas: Floquet Analysis, Synthesis, BFNs, and Active Array Systems, John Wiley \& Sons, Hoboken, NJ, USA, 2006.

[5] R. J. Mailloux, Electronically Scanned Array. Synthesis Lecture on Antennas, Morgan \& Claypool Publishers, Constantine Balanis, Ariz, USA, 2007.

[6] J. Drouet, M. Thevenot, R. Chantalat et al., "Global synthesis method for the optimization of multi feed EBG antennas," International Journal of Antennas and Propagation, vol. 2008, Article ID 790358, 6 pages, 2008.

[7] S. K. Sharma and L. Shafai, "Beam focusing properties of circular monopole array antenna on a finite ground plane," IEEE Transactions on Antennas and Propagation, vol. 53, no. 10, pp. 3406-3409, 2005.

[8] C. Phongcharoenpanich, S. Suriya, T. Lertwiriyaprapa, P. Ngamjanyaporn, and M. Krairiksh, "Analysis of circular array of monopole on the ground plane radiating linearly polarized conical beam for wireless LAN applications," in Proceedings of the 5th International Symposium on Antennas, Propagation and EM Theory, pp. 646-649, Beijing, China, August 2000.

[9] V. Volski and G. A. E. Vandenbosch, "Modelling of microstrip antennas on a finite ground plane using the 2D physical optics model," Microwave and Optical Technology Letters, vol. 40, no. 1, pp. 26-29, 2004.

[10] H. Jasik, Antenna Engineering Handbook, McGraw-Hill Book Company, New York, NY, USA, 1961.

[11] J. Huang and A. C. Densmore, "Microstrip Yagi array antenna for mobile satellite vehicle application," IEEE Transactions on Antennas and Propagation, vol. 39, no. 7, pp. 1024-1030, 1991.

[12] H. Yagi, "Beam transmission of the ultra short waves," IRE Proceedings, vol. 16, no. 6, pp. 715-740, 1928.

[13] C. A. Balanis, Antenna Theory. Analysis and Design, John Wiley \& Sons, New York, NY, USA, 1997.

[14] S. K. Padhi and M. E. Bialkowski, "Parametric study of a microstrip Yagi antenna," in Proceedings of the Asia-Pacific Microwave Conference, pp. 715-718, Sydney, NSW, Australia, December 2000. 

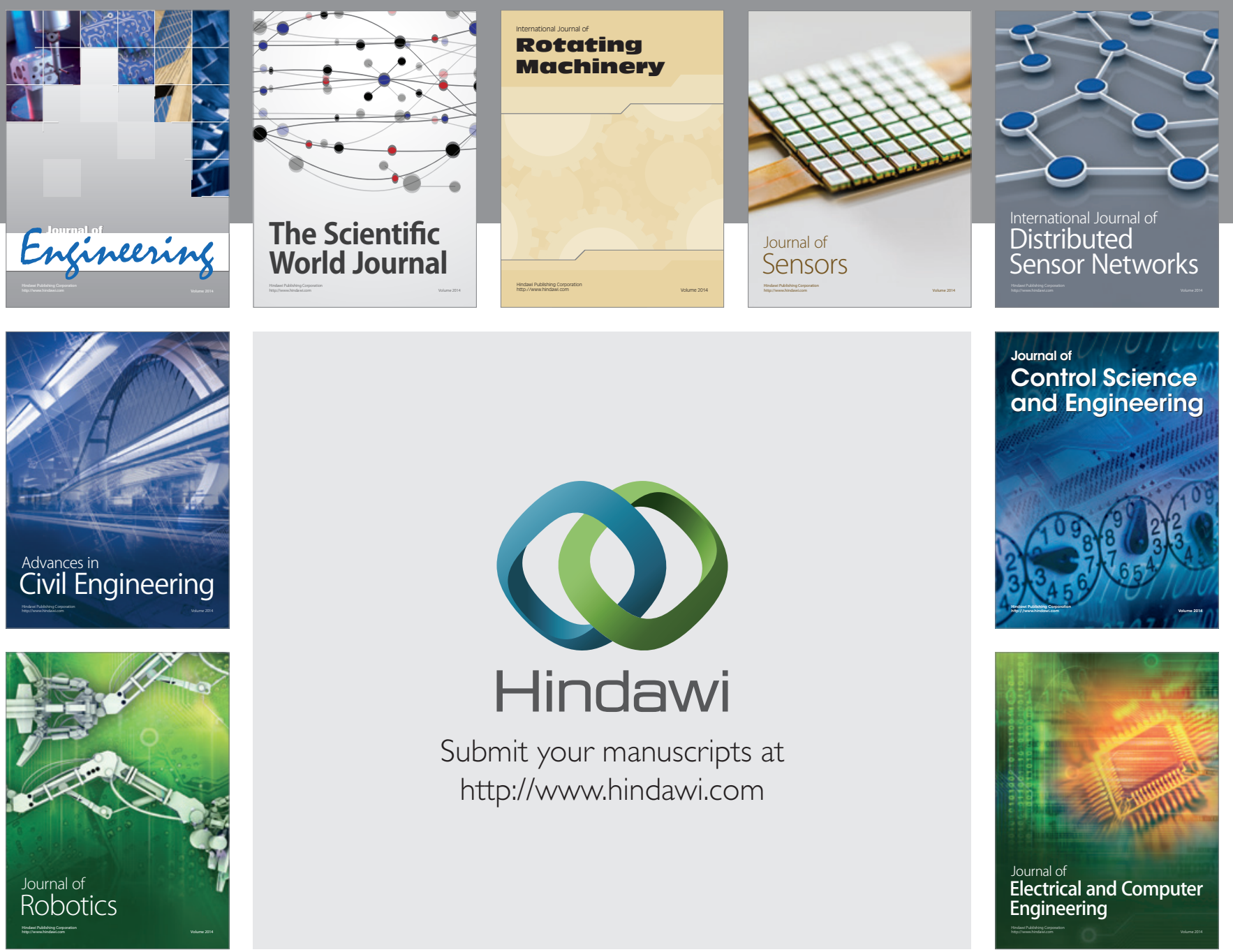

Submit your manuscripts at

http://www.hindawi.com
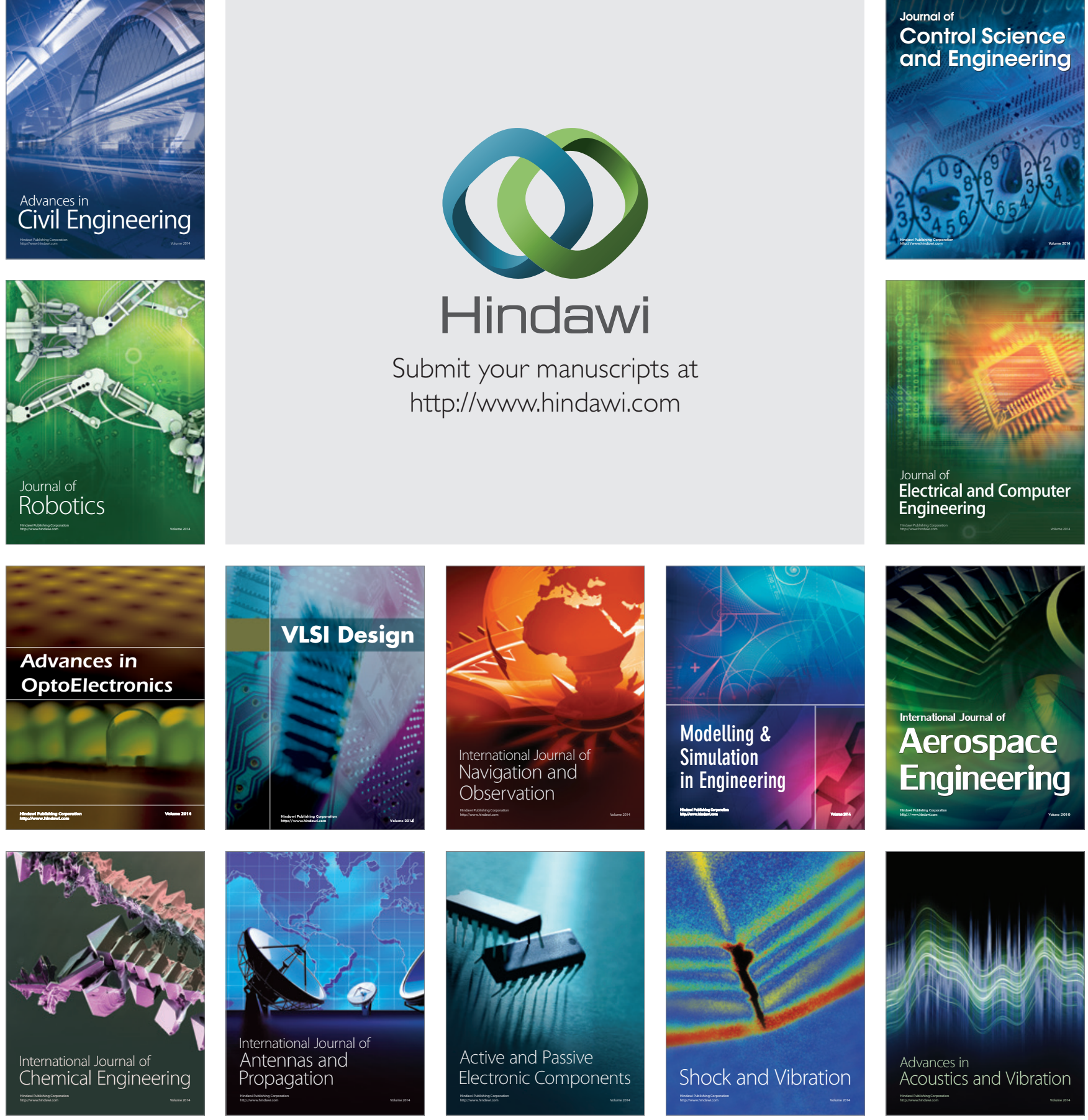